Published in final edited form as:

Female Pelvic Med Reconstr Surg. 2019 ; 25(2): 113-119. doi:10.1097/SPV.0000000000000681.

\title{
A 12-Month Clinical Durability of Effectiveness and Safety Evaluation of a Vaginal Bowel Control System for the Nonsurgical Treatment of Fecal Incontinence
}

\author{
Holly E. Richter, PhD, MD*, Gena Dunivan, MD ${ }^{\dagger}$, Heidi W. Brown, MD‡, Uduak Andy, MD§, \\ Keisha Y. Dyer, MD", Charles Rardin, MDI, Tristi Muir, MD**, Shane McNevin, MD ${ }^{\dagger \dagger}$, lan

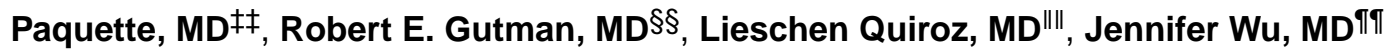 \\ *University of Alabama at Birmingham, Birmingham, AL \\ †University of New Mexico, Albuquerque, NM \\ ‡University of Wisconsin-Madison School of Medicine and Public Health, Madison, WI \\ §University of Pennsylvania, Philadelphia, PA \\ "Kaiser Permanente San Diego, San Diego, CA \\ "Brown University, Providence, RI \\ ${ }^{* *}$ Houston Methodist, Houston, TX \\ ${ }^{\dagger+}$ Providence Surgical Specialists, Providence, RI \\ $\ddagger \neq C h r i s t$ Hospital, Spokane, WA \\ $\S \S$ MedStar Washington Hospital Center/Georgetown University, Washington, DC \\ III'University of Oklahoma, Norman, OK \\ IfUniversity of North Carolina, Chapel Hill, NC.
}

\section{Abstract}

Objective: The aim of this study was to characterize clinical success, impact on quality of life, and durability up to 1 year in women with fecal incontinence (FI) responsive to an initial test period with a trial vaginal bowel control system.

Methods: This was a prospective open-label study in subjects with FI and successfully fit who underwent an initial 2-week trial period. Those achieving $50 \%$ or greater reduction in FI episodes were provided the long-term system. Primary outcome was success at 3 months defined as $50 \%$ or greater reduction in baseline FI episodes, also assessed at 6 and 12 months. Secondary outcomes included symptom impact measured with Fecal Incontinence Quality of Life scale, symptom

Correspondence: Holly E. Richter, PhD, MD, Division Urogynecology and Pelvic Reconstructive Surgery, University of Alabama at Birmingham, 1700 6th Ave South, Suite 10382, Birmingham, AL 35233. hrichter@uabmc.edu.

Clinical Trial Registration: (http://clinicaltrials.gov).

Supplemental digital contents are available for this article. Direct URL citations appear in the printed text and are provided in the HTML and PDF versions of this article on the journal's Web site (www.fpmrs.net). 
severity by the St Mark's (Vaizey) questionnaire, Patient Global Impression of Improvement, and satisfaction. Adverse events were collected. Primary analysis was intention to treat (ITT).

Results: Seventy-three subjects with baseline mean of $14.1 \pm 12.15$ FI episodes over 2 weeks entered the treatment period. Success rate at 3 months was $72.6 \%(53 / 73, P<0.0001)$; perprotocol, 84.1\% (53/63, $P<0.0001)$. Significant improvement in all Fecal Incontinence Quality of Life subscales and St Mark's questionnaire meeting minimally important differences was noted. Satisfaction was $91.7 \%, 89.7 \%$, and $94.4 \%$ at 3, 6, and 12 months, respectively; $77.4 \%, 77.6 \%$, and 79.6\% were very much/much better on the Patient Global Impression of Improvement at 3,6, and 12 months, respectively. Most common adverse event was vaginal wall injury, with most adverse events (90/134, 67\%) occurring during fitting period.

Conclusions: In women with successful fitting and initial treatment response, durable efficacy was seen at 3,6, and 12 months by objective and subjective measures, with favorable safety.

\section{Keywords}

fecal incontinence; quality of life; vaginal bowel control system

Fecal incontinence (FI) is a debilitating condition that negatively impacts quality of life, leads to social isolation, and increases the likelihood of institutionalization. ${ }^{1-3}$ Prevalence rates in community-dwelling women range from $12 \%$ to $25 \% .{ }^{4-6}$ First-line treatment for FI is behavioral therapy and there are limited other therapies available before more invasive surgical procedures are considered. Effective management may involve the use of multiple treatment modalities.

A vaginal bowel control (VBC) system (Eclipse System; Pelvalon, Sunnyvale, Calif) was designed to offer a low-risk, effective, and easily reversible treatment for FI. The system consists of a silicone-coated vaginal insert with posteriorly oriented balloon and detachable pump that reversibly deflects the rectovaginal septum and interrupts the passage of stool. A previous proof of efficacy and safety study revealed that at 1 month $78.7 \%$ of the intentionto-treat (ITT) cohort (95\% confidence interval [CI], 66\%-88\%; $P<0.0001$ ) met success criterion; $85.7 \%$ (95\% CI, 74\%-94\%, $P<0.0001$ ) met success criterion in per-protocol (PP) analysis, and $85.7 \%$ of participants considered their bowel symptoms "very much" or "much better." "There was significant improvement in all Fecal Incontinence Quality of Life (FIQOL) and Modified Manchester Health Questionnaire total and subscale scores, with attainment of minimally clinically important differences and no device-related serious adverse events.

The objective of this study was to characterize clinical effectiveness and safety of the VBC system in women successfully fit and who attained a $50 \%$ or greater reduction in FI episodes in an initial test diary. End points were assessed at 3 through 12 months compared with baseline. We anticipated that a similar effectiveness and safety profile would be demonstrated with long-term use. 


\section{MATERIALS AND METHODS}

Research approval was obtained from site-specific and other independent institutional review boards; all participants provided written informed consent. This was a prospective, open-label, 12-month clinical effectiveness trial of a VBC system in women with FI. Subjects were given a 2-week baseline bowel diary to determine study eligibility based on FI episodes. The diaries captured frequency, severity (staining, minor soiling, major soiling), consistency (Bristol Stool Scale), and any associated fecal urgency episodes. ${ }^{8}$ Only major (required an immediate change of undergarment, pad, or clothing) and minor (more than staining, but did not require an immediate change of undergarment, pad, or clothing) FI episodes were used for eligibility criteria and outcome assessments. Stool staining alone was not considered a leakage episode.

Subject eligibility included women 19 years or older, with a history of FI for at least 6 months and a minimum of 4 FI episodes during the 2-week baseline bowel diary evaluation period. Participants also had to undergo a successful evaluation and treatment with a trial VBC system, which was a similar but less durable version of the long-term system. Participant exclusion criteria consisted of the following: FI primarily due to chronic watery diarrhea unmanageable by medications or diet, concurrent medical conditions such as urinary or colorectal infections, presence of a rectovaginal fistula, tumor of genitourinary or colorectal origin, inflammatory bowel disease, chronic pain syndromes of the pelvis and/or anorectal origin, vaginal prolapse extending beyond the plane of the hymen, previous rectal or pelvic surgery within the last 12 months (24 months in the case of cancer), congenital anorectal malformation, significant urogenital atrophy, presence of an open wound or tear in the vagina or anus, pregnancy or subjects planning pregnancy in the next 5 months, and any other significant medical conditions that would interfere with study participation such as psychiatric or neurological disorders or active alcohol or drug abuse that would increase the subject's risks due to participation.

Study subjects provided demographic data and previous interventions for FI including dietary/behavioral modifications, medications, pelvic floor therapy with or without biofeedback, and surgical procedures. A baseline pelvic and rectal examination was performed that included an assessment for prolapse, urogenital atrophy, and rectal resting and squeeze pressures.

Eligible patients proceeded to VBC system fitting, working directly with the provider to determine the appropriate size and inflation pressure (chosen from 3 preset ranges). Three attempts at successful fitting were allowed. Once successfully fit, the subject was entered into the run-in phase of the study. Subjects were instructed on system usage and received inflation/deflation, insertion/removal, and cleaning instructions. Subjects meeting a 50\% reduction in FI episodes during the run-in phase were offered continued participation into the treatment period and comprised the intent-to-treat (ITT) population. These patients were given the more durable VBC system.

The treatment phase of the study consisted of intravaginal device use for 12 months. Participants completed treatment bowel diaries documenting the last 2 weeks of each 
assessment period at 3 months (primary outcome time-point), 6 months, and 12 months. Adverse events (AEs), defined as any untoward medical occurrence, unintended disease or injury, or untoward clinical signs (including abnormal laboratory findings), were documented, and relationship to the investigational device was assessed. Adverse event severity was rated as mild (transient and easily tolerated by the subject), moderate (causes the subject discomfort and interrupts her usual activities), or severe (causes considerable interference with the subject's usual activities; may be incapacitating and may require hospitalization). All AEs were adjudicated by the clinical events committee chair and medical monitor.

The primary outcome was the proportion of patients with a $50 \%$ or greater reduction in the mean number of FI episodes by bowel diary during the 3-month treatment compared with baseline. This outcome was also evaluated at 6 and 12 months. The primary efficacy end point was the proportion of subjects achieving a $50 \%$ or greater reduction in FI episodes, based on the ITT population, defined as all subjects who were successfully fitted with the trial system and met the test diary requirements. Subjects who did not complete the full 3month use or did not complete the treatment diary were classified as treatment failures in the ITT analysis. The null hypothesis $\left(\mathrm{H}_{0}\right)$ was that no more than $40 \%$ of women would have treatment success, and the alternative hypothesis $\left(\mathrm{H}_{\mathrm{A}}\right)$ was that more than $40 \%$ of women would attain treatment success. Additional efficacy and secondary outcome analyses were performed on the PP population, which included subjects with at least 3-month follow-up and no major protocol deviations and who had a valid bowel diary at 3-, 6-, and 12-month treatment periods.

Postintervention physical examination assessments and secondary outcome measures were collected at 3, 6, and 12 months to further assess objective physical findings and subjective measures of improvement. Symptom severity was evaluated using the validated St Mark's (Vaizey) questionnaire. ${ }^{9,10}$ The FIQOL ${ }^{11}$ questionnaire was also completed at all time points and includes subscales with scores ranging from 1 to 4 , except the Depression/Selfperception (range, 1-6). Higher scores corresponding to better quality of life, treatment satisfaction, and Patient Global Impression of Improvement (PGI-I) for bowel control on a scale of 1 (very much better) to 7 (very much worse) were also obtained. ${ }^{12}$ Adverse events were reported throughout the study. Postintervention assessments also included a pelvic examination to check for vaginal irritation or injury.

The sample size calculation was based on an estimated $67 \%$ response rate, calculated from an estimated $85.6 \%$ success rate among study completers and $21.3 \%$ dropout within 3 months in a previous study. ${ }^{7}$ With as few as 40 subjects, the analysis of the ITT population has greater than $90 \%$ power $(a=0.025)$ to detect a response rate greater than $40 \%$. Additional subjects were enrolled in order to ensure that a sufficient number of subjects were present in the ITT population.

Results from St Mark's (Vaizey) and FIQOL total and subscales scores, numbers of FI episodes reported during a 2 -week period, and continuous demographic and baseline variables were summarized using counts, means, and SDs. Changes from baseline in the questionnaire subscales, at 3,6, and 12 months, were evaluated using a repeated-measures 
analysis of the change from baseline, with the baseline subscale score used as a covariate, on the PP population. Changes in the number of FI episodes were similarly analyzed.

Responses to the PGI-I and the proportion of subjects reporting device satisfaction were also summarized. Proportions of subjects achieving $75 \%$ or greater reduction and $100 \%$ reduction (complete continence), and 50\% or less reduction were calculated. Incidence rates were reported for device-related adverse events. SAS 9.4 (SAS Institute, Cary, NC) was used for all analyses.

\section{RESULTS}

Participants were recruited and treated from May 2015 to March 2018. A total of 252 women consented to participation in the study, and flow through the study is shown in Figure 1. The majority of exclusions after consent were prefitting screen failures due to insufficient frequency of incontinence episodes on the 2-week baseline diary. Of 137 subjects who entered the fitting assessment, $85(62.0 \%)$ achieved a successful fit and underwent test diary screening. Seventy-three $(85.9 \%)$ of those subjects achieved at least a $50 \%$ reduction in FI episodes on their test diaries and continued participation in this 12month treatment study. These 73 subjects who successfully completed the fitting process with the VBC system and achieved at least a 50\% reduction in FI episodes in the test diary comprised the ITT population.

Relevant demographic and clinical characteristics of the ITT population are noted in Table 1. The sample was predominantly white and postmenopausal, with a mean age of 61 years; 37 $(50.7 \%)$ of 73 were sexually active within the last 6 months. At baseline, the mean \pm SD and median number of FI episodes per 2-week period were $14.1 \pm 12.15$ and 10 (range, 4-83), respectively. Almost half $(36 / 73,49.3 \%)$ had experienced FI symptoms for more than 5 years, and 71 (97.3\%) of 73 had attempted prior self-management strategies. Mean resting and squeeze Digital Rectal Examination Scoring System scores were $2.2 \pm 0.8$ (range, 1-4) and $2.1 \pm 1.1$ (range, $0-4$ ), corresponding to mildly decreased resting tone and fair increase but below normal squeeze tone. Other bowel-specific symptoms are noted in Table 1.

At 3 months, the success rate was $73 \%$ (95\% CI, 61\%-82\%; $\mathrm{n}=53 / 73 ; P<0.0001)$ in the ITT population and $84 \%(95 \% \mathrm{CI}, 73 \%-92 \% ; \mathrm{n}=53 / 63 ; P<0.0001)$ in the PP population. Success rates remained high at 6 and 12 months in the ITT population and improved to $90 \%$ to $94 \%$ in the PP population (Fig. 2). In the PP population, complete continence was achieved in 37\% (23/63), 49\% (28/57), and 46\% (25/54) at 3, 6, and 12 months, respectively. Mean incontinence episodes and St Mark's scores significantly decreased from baseline to all time points in the PP group, and FIQOL scores significantly increased (Fig. 3). Major FI episodes/2 weeks decreased from $5.0 \pm 6.03$ baseline to $1.2 \pm 1.88$ and $1.5 \pm 0.99$ at 3 and 12 months, respectively $(P<0.0001)$. Similarly, minor FI episodes $/ 2$ weeks decreased from $9.1 \pm 9.92$ baseline to $1.8 \pm 2.70$ and $1.3 \pm 2.29$ at 3 and 12 months, respectively ( $P<$ 0.0001 ). The FIQOL scores significantly improved in all subscales as well (see Appendix, Supplemental Digital Content 1, http://links.lww.com/FPMRS/A71).

More than $90 \%$ of participants in the PP population reported that their symptoms improved on the PGI-I at 3 months, and this increase was sustained at 12 months (Fig. 4), with more

Female Pelvic Med Reconstr Surg. Author manuscript; available in PMC 2019 July 26. 
than $75 \%$ reporting that their symptoms were very much or much better at each time point. Satisfaction remained high in this group at $91.8 \%$ (56/61), 89.7\% (52/58), and $94.4 \%$ (51/54) at the 3-, 6-, and 12-month time points, respectively.

There were no serious device-related adverse events experienced by subjects involved in any portion of the study; the majority of adverse events occurred during the fitting period, with $n$ $=62$ experiencing mild or moderate AEs related to the device. Of 73 subjects in the treatment period, $\mathrm{n}=28$ experienced mild or moderate AEs related to the device. The majority of vaginal wall injuries (94\% in the treatment period) were mild, and none resulted in study discontinuation. During the fitting period, $80 \%$ of vaginal wall injuries were mild. Adverse events are noted in Table 2.

\section{DISCUSSION}

The current study shows that after the VBC system is successfully fit it is a highly effective and durable nonsurgical treatment option for FI. This study validates the prior work demonstrating safety and efficacy of the VBC system at 1 month and demonstrates similar effectiveness and safety profile by both subjective and objective measures at 3,6 , and 12 months. In addition, it was found that nearly half (46\%) of PP subjects had complete continence, and more than $80 \%$ of remaining PP subjects had more than a $75 \%$ reduction in FI symptoms at 12 months.

In 2016, the Agency for Healthcare Research and Quality (AHRQ) conducted an extensive systematic review on FI treatments and determined that there was little evidence for treatment effectiveness beyond 3 to 6 months and that existing evidence available was of low strength. ${ }^{13}$ Additionally, they concluded there was not enough evidence to support one treatment over another and that surgical interventions carry higher risk. ${ }^{14}$ These findings emphasize the importance of this nonsurgical, longer-term, safety, and efficacy trial.

Studies comparing other treatments for FI, in particular, sacral neuromodulation, anal bulking agents, and anal plugs, typically utilize the outcome measure of $50 \%$ or greater improvement in FI. This trial was designed to allow for similar comparisons between treatments with our primary outcome of $50 \%$ or greater improvement in FI, and high success rates at 3 months, which were maintained through 1 year. In an evaluation of a new singleuse, disposable anal insert (Renew Insert), $62 \%$ of subjects achieved a $50 \%$ or greater reduction in FI episodes in the intent-to-treat protocol. ${ }^{15}$ For anal bulking agents, the results are relatively disappointing. In a randomized trial, $52 \%$ of the dextranomer in hyaluronic acid group had treatment success ( $>50 \%$ reduction in FI episodes at 6 months); however, there was a notable placebo effect as $32 \%$ of sham injection group had treatment success. ${ }^{16}$ For sacral nerve stimulation, a multicenter study reported that among patients who went on to have a full implant $83 \%$ had treatment success on per-protocol analysis ( $250 \%$ improvement in FI) at 12 months, and $41 \%$ had complete continence. In the worst-case analysis that assumed failure if 12 -month data were missing, success was $73 \% .{ }^{17}$ These findings are comparable to our PP success rate at $94 \%$ and $46 \%$ with complete continence at 12 months. 
Further validation of the current results is the improvement in patient-reported outcomes of FI severity (St Mark's score), quality of life (FIQOL), patient impression of improvement (PGI-I), and satisfaction. Notably, the current data met the minimally important difference for both the St Mark's score (-5) and the FIQOL (1.1-1.2). ${ }^{10,18}$ Overall, the findings from this longer-term study are comparable to the results of the original safety and efficacy LivSure for Fecal Incontinence trial with PP success of 85.7\% (48/56, $P<0.001), 41 \%$ with complete continence at 1 month, and no serious adverse events. ${ }^{7}$

The highly efficacious and durable results seen in this study are likely due to the mechanical mechanism of the device. This device impacts the dynamic opening and closing of the anorectal canal to help replicate normal functioning of the rectum and may help patients by improving sensation. In the initial short-term study, this device was associated with a reduction in bowel movement frequency, less fecal urgency, increased solid consistency, and improved evacuation. ${ }^{19}$ In the current study, the fitting process and run-in phase provided opportunities to identify patients with improvement in symptoms who were likely to be comfortable with the daily maintenance of the device, similar to other vaginal inserts such as pessaries. These visits were optimal times to discuss expectations and to ensure proper usage and cleaning of the device. Overall retention rates were high (74\%, 54/73 completed all 12 months), and $98 \%$ (53/54) of patients opted to continue the device after the 12-month end of the study. The ease and comfort of device use likely improved with consistent use as with any new task or treatment regimen. Additionally, with any patient-managed therapy, compliance is particularly important and noted retention rates (74\%) were reasonable at 1 year. In fact, when all subjects who discontinued the study are categorized as treatment failures (ITT analysis), the rate of success at 1 year in this study is comparable to that of sacral neuromodulation, a more invasive surgical procedure (70\% vs $73 \%$, respectively). ${ }^{17}$

A limitation of this study is that all women were not able to be successfully fit, similar to a vaginal pessary. Yet, $62 \%$ (85/137) of the women underwent a successful fitting, an improvement from the $54.5 \%$ in the initial study, which may be related to the provider learning curve. ${ }^{7}$ In addition, shorter vaginal length and prior prolapse surgery may decrease the likelihood of successful fitting. ${ }^{20}$ Another limitation is that the current study population had relatively severe FI, with a minimum of 4 major incontinence episodes over 2 weeks, and therefore, the results may not be generalizable to those with less severe FI or staining only. However, it is reassuring that an improvement was noted in both major and minor incontinence episodes. Because this was a safety and efficacy trial with strict inclusion/ exclusion criteria, external validity, or generalizability, may be limited to populations different from the current participants. However, one could note that in subjects with more or fewer FI episodes the described trial period is straightforward and the device risk is low. Therefore, offering this treatment approach would not be unreasonable in the algorithm of clinical practice for women with FI. Finally, there was no control group to control for placebo effect, but with sustained benefit noted over 12 months with both objective and subjective assessments using a low-risk device, we feel that these results are valid.

A major strength of this study is that it was conducted across multiple clinical sites across the country and that the study population was fairly diverse. These longer-term results confirm the initial short-term findings of the original study of this VBC system, highlighting 
the durability of this novel device. ${ }^{7}$ Furthermore, improvement in objective data with reduction in FI episodes was noted as well as multiple patient-reported outcomes using validated symptom-specific severity and quality-of-life measures.

In conclusion, the VBC device is a safe, effective, and durable treatment option for women suffering from FI and could be utilized early in the FI treatment algorithm concomitantly with other treatment options. This prospective, multicenter study provides critically important longer-term results of this new system. Future efforts should focus on long-term, comparative studies ${ }^{21}$ which include the evaluation of this safe and effective VBC system.

\section{Supplementary Material}

Refer to Web version on PubMed Central for supplementary material.

\section{ACKNOWLEDGMENTS}

The authors thank Madhulika Varma, MD; Catherine Ann Matthews, MD; and Miki Takase Sanchez, MD.

Each author received grant support from Pelvalon, Inc. Dr Richter has declared no conflicts of interest.

\section{REFERENCES}

1. Meyer I, Richter HE. Impact of fecal incontinence and its treatment on quality of life in women. Womens Health (Lond) 2015;2:225-238.

2. Yip SO, Dick MA, McPencow AM, et al. The association between urinary and fecal incontinence and social isolation in older women. Am J Obstet Gynecol 2013;2:146.e1-146.e7.

3. Shah BJ, Chokhavatia S, Rose S. Fecal incontinence in the elderly: FAQ. Am J Gastroenterol 2012;11:1635-1646.

4. Varma MG, Brown JS, Creasman JM, et al. Fecal incontinence in females older than aged 40 years: who is at risk? Dis Colon Rectum 2006;49:841-851. [PubMed: 16741640]

5. Bharucha AE, Zinsmeister AR, Locke GR, et al. Prevalence and burden of fecal incontinence: a population-based study in women. Gastroenterology 2005;129:42-49. [PubMed: 16012933]

6. Brown HW, Wexner SD, Lukacz ES. Factors associated with care seeking among women with accidental bowel leakage. Female Pelvic Med Reconstr Surg 2013;19:66-71. [PubMed: 23442502]

7. Richter HE, Matthews CA, Muir T, et al. A vaginal bowel-control system for the treatment of fecal incontinence. Obstet Gynecol 2015;125:540-547. [PubMed: 25730213]

8. Lewis SJ, Heaton KW. Stool form scale as a useful guide to intestinal transit time. Scand J Gastroenterol 1997;32:920-924. [PubMed: 9299672]

9. Vaizey CJ, Carapeti E, Cahill JA, et al. Prospective comparison of faecal incontinence grading systems. Gut 1999;44:77-80. [PubMed: 9862829]

10. Bols EM, Hendriks EJ, Deutekom M, et al. Inconclusive psychometric properties of the Vaizey score in fecally incontinent patients: a prospective cohort study. Neurourol Urodyn 2010;29:370377. [PubMed: 19634170]

11. Rockwood TH, Church JM, Fleshman JW, et al. Fecal incontinence quality of life scale: quality of life instrument for patients with fecal incontinence. Dis Colon Rectum 2000;43:9-16. [PubMed: 10813117]

12. Yalcin I, Bump RC. Validation of two global impression questionnaires for incontinence. Am J Obstet Gynecol 2003;189:98-101. [PubMed: 12861145]

13. Forte ML, Andrade KE, Butler M, et al. Treatments for Fecal Incontinence. Rockville MD: Agency for Healthcare Research and Quality (US); 2016. Report No:15 (16)-EHC037-EF. AHRQ Comparative Effectiveness Reviews. 
14. Forte ML, Andrade KE, Lowry AC, et al. Systematic review of surgical treatments for fecal incontinence. Dis Colon Rectum 2016;59:443-469. [PubMed: 27050607]

15. Lukacz ES, Segall MM, Wexner SD. Evaluation of an anal insert device for the conservative management of fecal incontinence. Dis Colon Rectum 2015;58:892-898. [PubMed: 26252852]

16. Graf W, Mellgren A, Matzel KE, et al. Efficacy of dextranomer in stablised hyaluronic acid for treatment of faecal incontinence: a randomised, sham-controlled trial. Lancet 2011;377:997-1003. [PubMed: 21420555]

17. Wexner SD, Coller JA, Devroede G, et al. Sacral nerve stimulation for fecal incontinence: results of a 120-patient prospective multicenter study. Ann Surg 2010;251:441-449. [PubMed: 20160636]

18. Bols EM, Hendriks EJ, Berghmans LC, et al. Responsiveness and interpretability of incontinence severity scores and FIQL in patients with fecal incontinence: a secondary analysis from a randomized controlled trials. Int Urogynecol J 2013;24:469-478. [PubMed: 22806487]

19. Varma MG, Matthews CA, Muir T, et al. Impact of a novel vaginal bowel control system on bowel function. Dis Colon Rectum 2016;59:127-131. [PubMed: 26734971]

20. Matthews CA, Varma MG, Takase-Sanchez MM, et al. Characteristics associated with successful fitting of a vaginal bowel control system for fecal incontinence. Female Pelvic Med Reconstr Surg 2016;22:359-363. [PubMed: 27564386]

21. Whitehead WE, Rao SS, Lowry A, et al. Treatment of fecal incontinence: state of the science summary for the National Institute of Diabetes and Digestive and Kidney Diseases workshop. Am J Gastroenterol 2015;110:138-146 quiz 47. [PubMed: 25331348] 


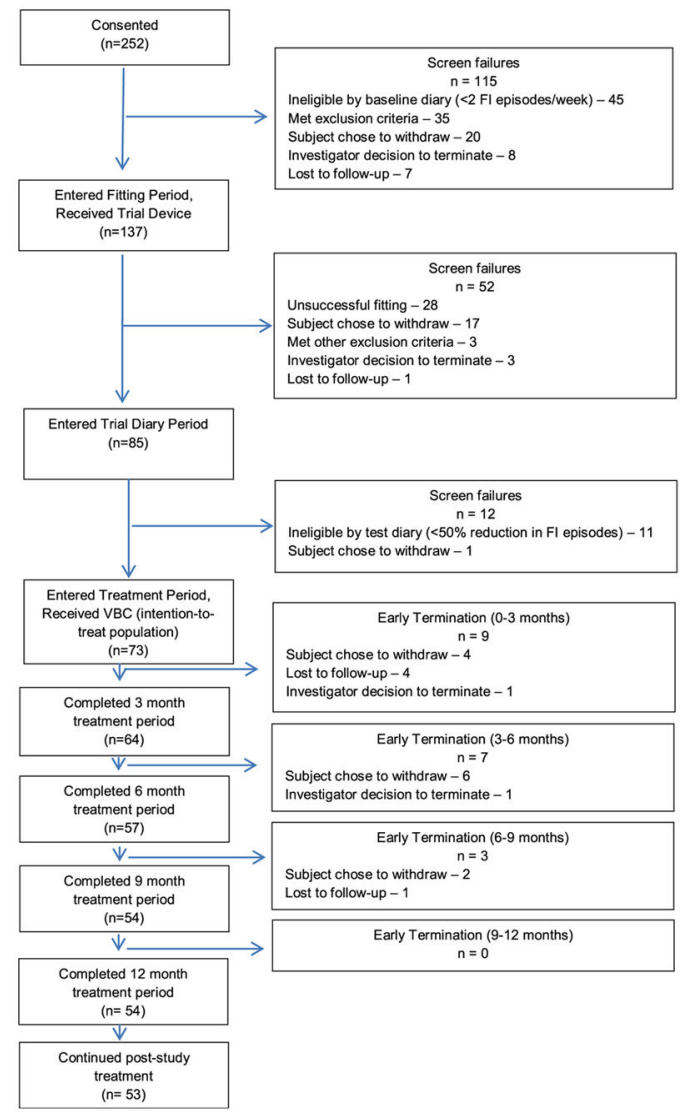

FIGURE 1.

Patient flow. 


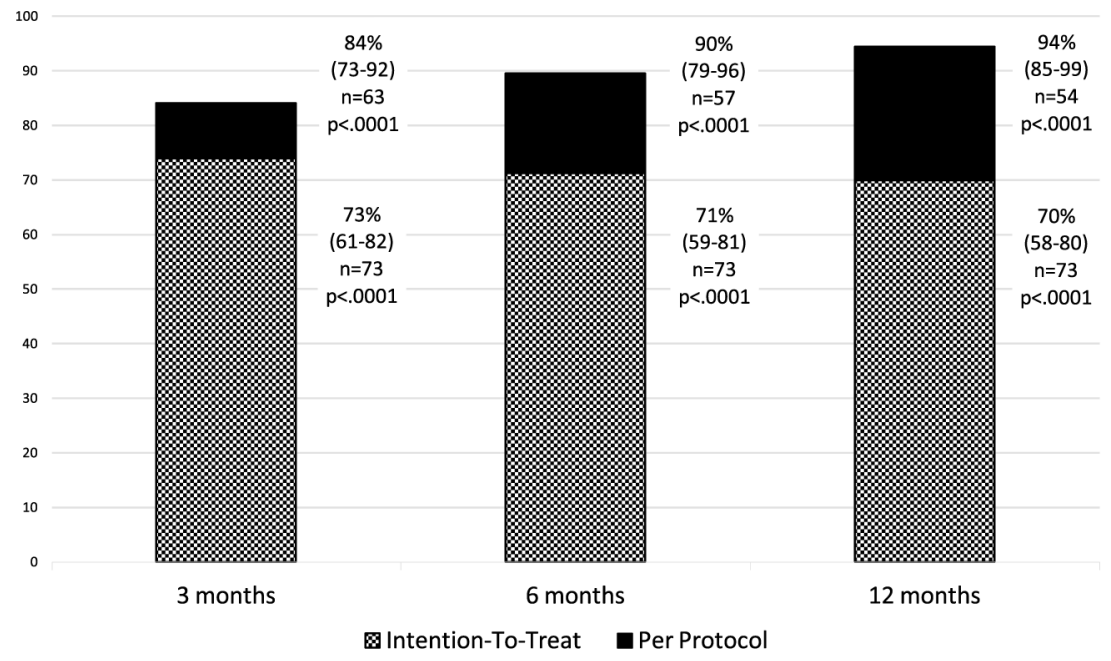

Legend: Values displayed are percentages of participants in each group with at least $50 \%$ reduction in frequency of FI episodes per 2 week period with $95 \%$ Clopper-Pearson confidence intervals; p-values reflect exact binomial test comparing these percentages to $40 \%$.

FIGURE 2.

Percentage of VBC system users with at least $50 \%$ reduction in number of FI episodes at 3, 6, and 12 months of use in ITT and PP populations. 


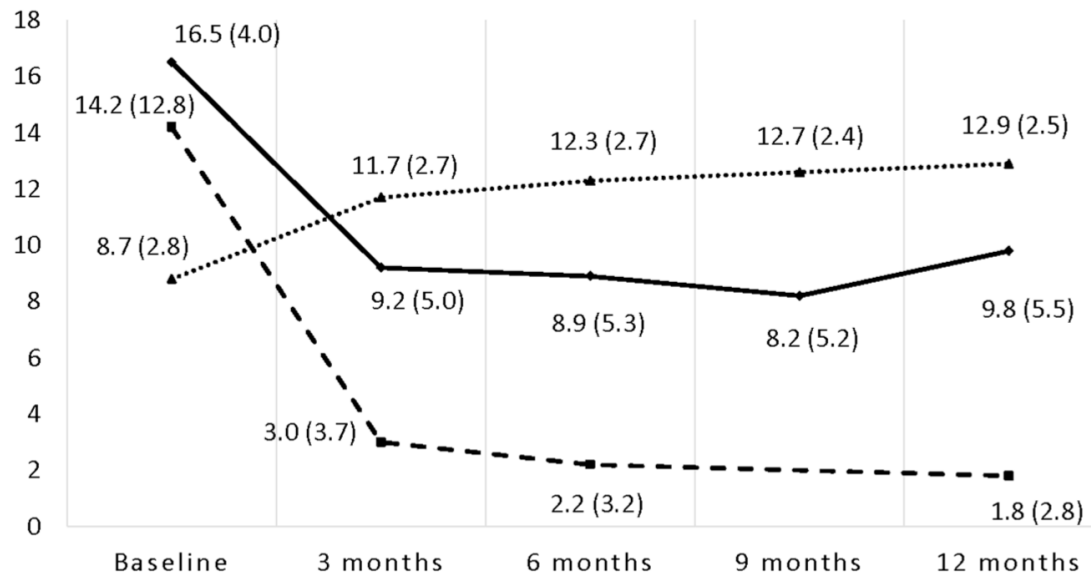

$\longrightarrow$ Mean (SD) St. Mark's Score

- - Mean (SD) Fl episodes/2 weeks

........ Mean (SD) FIQOL score

* All data points differ from baseline with p-values of $<.0001$. FIQOL $=$ Fecal Incontinence Quality of Life; (SD) $=$ Standard Deviation

FIGURE 3.

Fecal incontinence severity, frequency, and quality of life in PP population over time. 


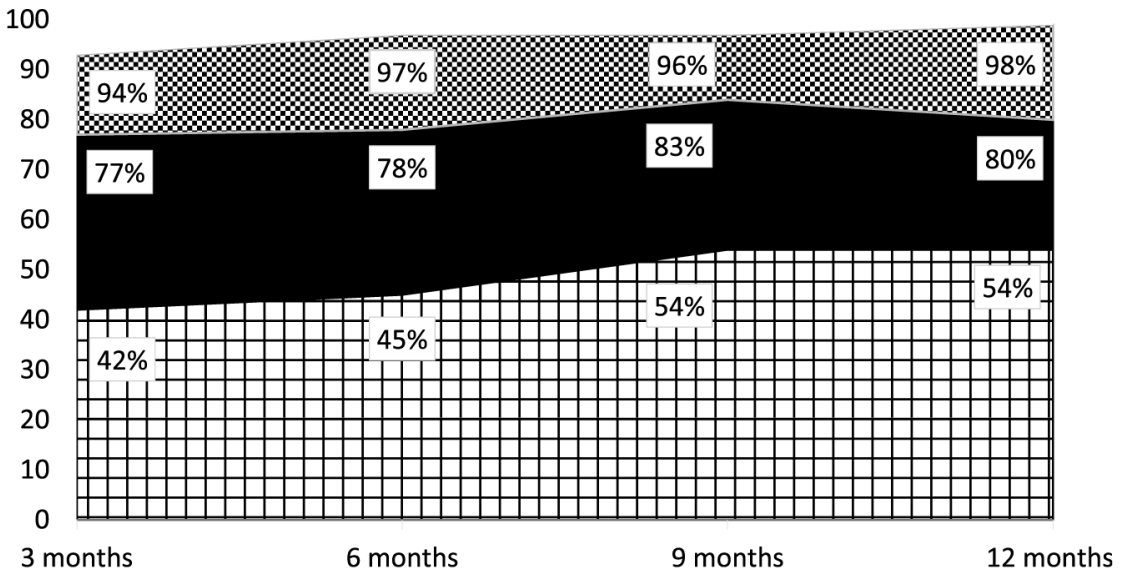

$\square$ Very much better $\square$ Much better $\mathbf{Q}$ A little better

FIGURE 4.

Patient Global Impression of Improvement in PP population. 
TABLE 1.

Baseline Demographic, Clinical, and FI Characteristics $(n=73)$

\begin{tabular}{|c|c|}
\hline & n $(\%)$ \\
\hline \multicolumn{2}{|l|}{ Demographic characteristics } \\
\hline Age, mean $\pm \mathrm{SD}$ (range), y & $61.3 \pm 11.51(24-94)$ \\
\hline \multicolumn{2}{|l|}{ Ethnic origin } \\
\hline White & $51(69.9 \%)$ \\
\hline Black & $17(23.3 \%)$ \\
\hline Hispanic or Latino & $5(6.8 \%)$ \\
\hline \multicolumn{2}{|l|}{ Clinical characteristics } \\
\hline \multicolumn{2}{|l|}{ Body mass index, $\mathrm{kg} / \mathrm{m}^{2}$} \\
\hline$<18.5$ & $1(1.4 \%)$ \\
\hline 18.5 to $<25$ & $23(31.5 \%)$ \\
\hline 25 to $<30$ & $27(37.0 \%)$ \\
\hline 230.0 & $22(30.1 \%)$ \\
\hline \multicolumn{2}{|l|}{ Previous surgeries/treatments } \\
\hline Hysterectomy & $30(41.1 \%)$ \\
\hline Prior prolapse surgery (excluding hysterectomy) & $10(13.7 \%)$ \\
\hline Prior urinary incontinence surgery & $10(13.7 \%)$ \\
\hline Gallbladder removal (cholecystectomy) & $9(12.3 \%)$ \\
\hline Fistula repair & $1(1.4 \%)$ \\
\hline None of these & $28(38.4 \%)$ \\
\hline \multicolumn{2}{|l|}{ Sexual activity within past 6 mo } \\
\hline Yes & $37(50.7 \%)$ \\
\hline \multicolumn{2}{|l|}{ Parity } \\
\hline No. vaginal births, mean \pm SD & $2.2 \pm 1.1$ \\
\hline No. vaginal births, median (range) & $2.0(0-6)$ \\
\hline Menopause status, self-reported & 73 \\
\hline Premenopause & $9(12.3 \%)$ \\
\hline Perimenopause & $4(5.5 \%)$ \\
\hline Postmenopause & $60(82.2 \%)$ \\
\hline \multicolumn{2}{|l|}{ Lower urinary tract and bowel conditions } \\
\hline Urinary incontinence & $22(30.1 \%)$ \\
\hline Overactive bladder (urinary frequency) & $34(46.6 \%)$ \\
\hline Irritable bowel syndrome & $15(20.5 \%)$ \\
\hline \multicolumn{2}{|l|}{ FI characteristics } \\
\hline FI type * & 66 \\
\hline Urge-predominant & $16(24.2 \%)$ \\
\hline Passive-predominant & $29(43.9 \%)$ \\
\hline Both (passive-urge) & $21(31.8 \%)$ \\
\hline Duration of symptoms & 73 \\
\hline $6 \mathrm{mo}$ to $5 \mathrm{y}$ & $37(50.7 \%)$ \\
\hline
\end{tabular}




\begin{tabular}{|c|c|c|c|c|c|c|c|}
\hline & \multicolumn{7}{|c|}{ n $(\%)$} \\
\hline$>5 \mathrm{y}$ & \multicolumn{7}{|c|}{$36(49.3 \%)$} \\
\hline \multicolumn{8}{|l|}{ Frequency } \\
\hline No. FI episodes/2-wk period, mean \pm SD & \multicolumn{7}{|c|}{$14.1 \pm 12.15$} \\
\hline No. FI episodes/2-wk period, median (range) & \multicolumn{7}{|c|}{$10.0(4-83)$} \\
\hline Major FI episodes/2-wk period, mean \pm SD & \multicolumn{7}{|c|}{$5.0 \pm 6.03$} \\
\hline Minor FI episodes/ 2-wk period, mean \pm SD & \multicolumn{7}{|c|}{$9.1 \pm 9.92$} \\
\hline Subjects with fecal urgency ${ }^{\dagger}$ & \multicolumn{7}{|c|}{$53(72.6 \%)$} \\
\hline Stool consistency-Bristol Stool Scale, mode & \multicolumn{7}{|c|}{4} \\
\hline \multirow[t]{2}{*}{ By patient Bristol proportions } & 1 & 2 & 3 & 4 & 5 & 6 & 7 \\
\hline & $6 \%$ & $4 \%$ & $13 \%$ & $28 \%$ & $20 \%$ & $22 \%$ & $9 \%$ \\
\hline $\begin{array}{l}\text { Daily stool frequency (per bowel diary, including normal bowel movement, major, minor } \\
\text { soiling), mean } \pm \text { SD }\end{array}$ & \multicolumn{7}{|c|}{$2.2 \pm 1.24$} \\
\hline \multicolumn{8}{|l|}{ Previous treatments for FI } \\
\hline Supervised PT exercises & \multicolumn{7}{|c|}{$17(23.3 \%)$} \\
\hline Biofeedback & \multicolumn{7}{|c|}{$13(17.8 \%)$} \\
\hline Surgery (sphincteroplasty, graciloplasty) & \multicolumn{7}{|c|}{$10(13.7 \%)$} \\
\hline Sacral nerve stimulation & \multicolumn{7}{|c|}{$8(11.0 \%)$} \\
\hline Injectable bulking agents & \multicolumn{7}{|c|}{$5(6.8 \%)$} \\
\hline \multicolumn{8}{|c|}{$\begin{array}{l}\text { Subjects were classified as urge-predominant, passive-predominant, or both if they reported "at least some of the time" to "I can't hold my bowel } \\
\text { novement long enough to get to the bathroom," "I leak stool without even knowing it," or both on FIQOL, respectively. Participants reporting "a } \\
\text { ittle" or "none" to both questions were excluded. }\end{array}$} \\
\hline \multicolumn{8}{|c|}{$\begin{array}{l}\text { 'Subjects were classified as having fecal urgency if } 25 \% \text { or greater bowel movements (includes normal bowel movements, minor and major soiling } \\
\text { episodes; excludes staining) were marked as "urgent" on bowel diary. }\end{array}$} \\
\hline
\end{tabular}




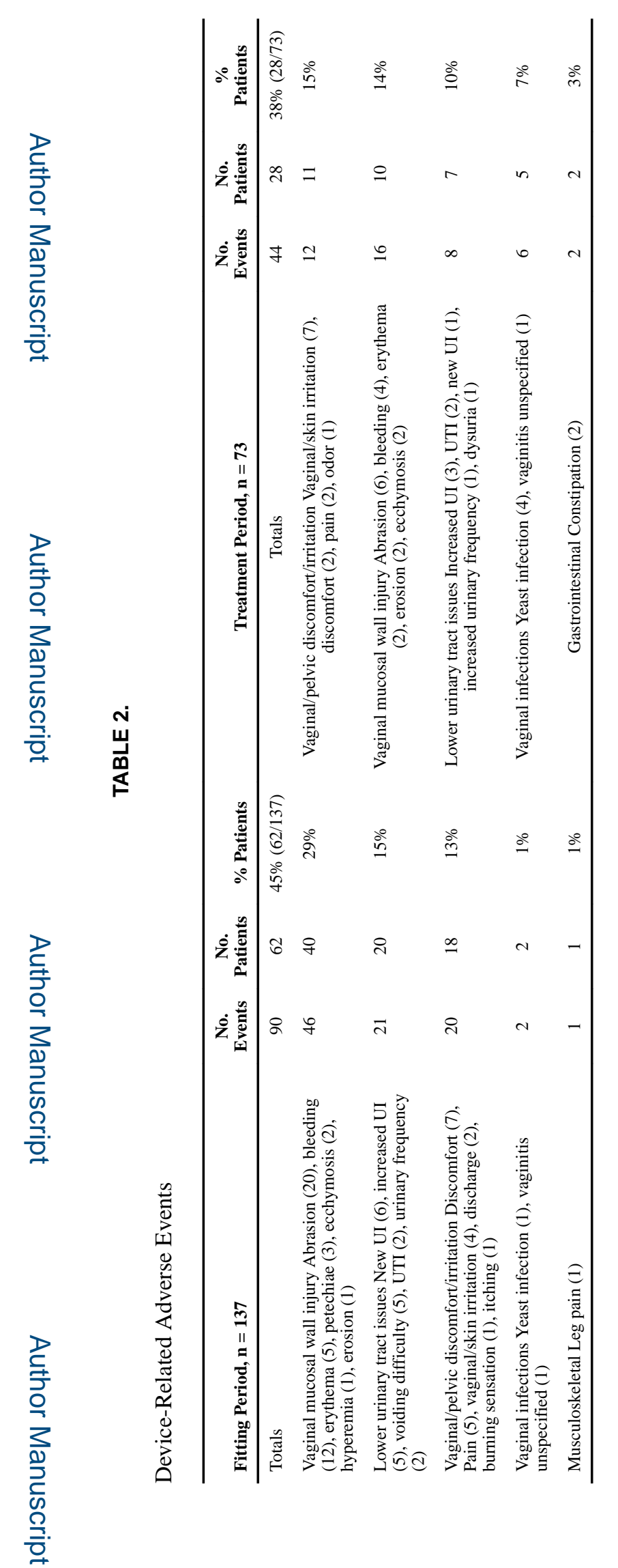

Female Pelvic Med Reconstr Surg. Author manuscript; available in PMC 2019 July 26. 